\title{
A ATENÇÃO PRIMÁRIA EM SAÚDE NA AGENDA DA ORGANIZAÇÃO PAN-AMERICANA DE SAÚDE NOS ANOS 2000
}

\author{
PRIMARY HEALTH CARE IN THE AGENDA OF THE PAN AMERICAN HEALTH ORGANIZATION \\ IN THE 2000s
}

\section{LA ATENCIÓN PRIMARIA DE LA SALUD EN LA AGENDA DE LA ORGANIZACIÓN PANAMERICANA DE LA SALUD EN LOS AÑOS 2000}

\author{
Alessandra Camargo da Silveira ${ }^{1}$ \\ Cristiani Vieira Machado ${ }^{2}$ \\ Gustavo Correa Matta ${ }^{3}$
}

Resumo O artigo aborda a agenda da Organização Pan-Americana da Saúde para a atenção primária em saúde e suas possíveis implicações na proteção social na América Latina nos anos 2000. O estudo, de natureza exploratória, partiu da literatura sobre proteção social e compreendeu como estratégias metodológicas a revisão bibliográfica e a análise documental, cujo referencial técnico foi a análise de retórica. A pesquisa procurou identificar as concepções e significados subjacentes à proposta de 'atenção primária em saúde renovada' no que concerne à perspectiva da proteção social, considerando três aspectos: a população-alvo (universal ou focalizada), o escopo (abrangente ou restrito) e a forma de organização dos serviços (relações público-privadas). Os resultados indicam que a agenda política da Organização Pan-Americana da Saúde apresenta historicamente continuidades e descontinuidades. O movimento de renovação da atenção primária em saúde expressa uma inflexão importante nessa agenda, pois sugere uma visão da atenção primária em saúde como estratégia de reestruturação dos sistemas nacionais de saúde, para além da abordagem programática. Porém, no que se refere à proteção social, as propostas apresentadas parecem compatíveis com diferentes arranjos de sistemas de saúde no que tange à concepção sobre o universalismo, o escopo das ações e as parcerias público-privadas.

Palavras-chave atenção primária em saúde; Organização Pan-Americana da Saúde; política social; serviços de saúde; Américas.
Abstract The article discusses the agenda of the Pan American Health Organization for primary health care and its implications for social protection in Latin America in the 2000s. Exploratory in nature, the study was based on the literature concerning social protection and included the review of the literature and documental analysis as methodological strategies, the technical reference for which was rhetoric analysis. The research sought to identify the concepts and meanings underlying the proposal for 'renewed health in primary care' insofar as the perspective of social protection is concerned, considering three aspects: target population (universal or targeted), scope (comprehensive or limited), and the way the services are organized (public-private relations). Results indicate that, historically, the Pan American Health Organization's political agenda has had continuities and discontinuities. The movement for the renewal of primary health care expresses an important inflection on this agenda, as it suggests a vision for primary health care as a restructuring strategy for the national health systems, in addition to the programmatic approach. However, concerning social protection, the proposals that have been made seem to be compatible with different health system arrangements with regard to the concept of universalism, action scope, and public-private partnerships.

Keywords primary health care; Pan American Health Organization; social policy; health services; Americas. 


\section{Introdução}

A construção de sistemas de saúde nacionais representa, ao longo da história, importante capítulo na constituição dos modernos sistemas de proteção social e na concepção da saúde como um direito. E a política de saúde resulta da interação de opções estatais, governamentais e sociais, sendo também influenciada por interesses de mercado.

No plano internacional, a Organização Mundial da Saúde (OMS) e o Fundo das Nações Unidas para a Infância (Unicef) desempenharam papel importante na difusão do conceito de atenção primária em saúde (APS). A I Conferência Internacional sobre Cuidados Primários de Saúde, convocada pela OMS juntamente com o Unicef, realizada em 1978 em Alma-Ata, é considerada um marco histórico na discussão da atenção primária em saúde. Nessa conferência, os cuidados primários em saúde foram propostos como estratégia para alcançar a meta "Saúde para todos no ano 2000" e apontados como componente fundamental de um sistema de saúde eficaz (Cueto, 2007).

Desse modo, a APS passou a ocupar espaço relevante no debate sobre as políticas de saúde, tornando-se elemento central nas reformas setoriais. Contudo, o termo APS adquiriu historicamente diferentes concepções, interpretações e finalidades e, com isso, assumiu distintos papéis na proteção social e nos sistemas nacionais de saúde.

As agências internacionais foram atores fundamentais na difusão de propostas relativas à APS para os países em desenvolvimento, principalmente a partir da década de 1980, com diferentes perspectivas. Enquanto a OMS manteve seu posicionamento a favor da APS mais abrangente, o Unicef adotou uma concepção mais seletiva. Já nos anos 1990, em um contexto de reforma dos Estados e difusão de ideias neoliberais, o Banco Mundial passou a se destacar. Assim, historicamente, o conceito de APS mostra-se multifacetado. Todavia, no contexto das reformas dos Estados nacionais, com predomínio da agenda neoliberal, na década de 1990, o Banco Mundial destacou-se na difusão de propostas de reformas dos sistemas de saúde baseadas na focalização e na adoção de serviços restritos (Mattos, 2001), fragilizando o protagonismo anterior da OMS e da Organização Pan-Americana da Saúde (Opas) na proposição de diretrizes para a saúde na região das Américas (Costa, 1998). A implantação de medidas de ajuste estrutural, acoplada à contenção de gastos públicos e ao predomínio de políticas sociais focalizadas, fomentou o aumento da pobreza e das desigualdades sociais, o que trouxe implicações para a proteção social e prejuízos às condições de saúde das populações latino-americanas.

A Opas é a mais antiga agência internacional atuante nas Américas e tem na cooperação técnica a principal estratégia para influenciar seus países-membros. Historicamente, em mais de um século de atuação, a Opas 
exerceu influência no âmbito da saúde internacional nessas regiões, por meio de diferentes propostas - inicialmente, com o fomento e o desenvolvimento de programas de saneamento de portos; e, posteriormente, com iniciativas como os programas de imunização e erradicação de doenças, entre outras (Lima, 2002). Outro tema tradicional da atuação da Opas nas últimas décadas diz respeito à formação e à capacitação de profissionais de saúde.

Nos anos 2000, a Opas propôs a retomada do legado da Conferência de Alma-Ata, por meio do movimento "Renovação da Atenção Primária em Saúde nas Américas". A agência passou a defender que renovar a APS não significaria um ajuste à conjuntura, mas concebê-la como estratégia estruturante dos sistemas de saúde com vistas à sua organização e coordenação para que esses sistemas sejam mais efetivos e eficientes e, assim, reduzam as iniquidades em saúde (Organização Pan-Americana da Saúde e Organização Mundial da Saúde, 2007).

Este artigo objetiva analisar a agenda da Opas para a APS, construída nos anos 2000, e suas possíveis implicações para a proteção social na América Latina. Além desta introdução, o texto compreende três itens. O primeiro deles discute as relações entre as distintas concepções de APS e o caráter da proteção social em saúde; o segundo resume a metodologia; e o terceiro item apresenta os principais resultados de pesquisa realizada sobre a agenda da Opas para a APS, interpretados à luz da literatura sobre o tema, considerando os possíveis significados e as implicações das propostas recentes da Opas relativas à APS em termos da proteção social em saúde nos países latino-americanos.

\section{Atenção primária em saúde e proteção social}

A conformação dos sistemas nacionais de saúde é um capítulo importante na constituição dos modernos sistemas de proteção social. Tais sistemas expressam três importantes dimensões: a saúde como direito social; a dimensão política da saúde, visto que a configuração das políticas se relaciona com o grau de pressão social expresso nas arenas decisórias setoriais e extrassetoriais; e a saúde como um espaço de acumulação de capital, uma vez que o setor envolve vultosa quantia de recursos e, ainda, a operação de diferentes tipos de mercados (Freeman e Moran, 2002; Elias e Viana, 2007).

O papel da APS nas políticas de proteção social pode ser analisado com base em três elementos centrais: o escopo dos serviços ofertados, se abrangente ou restrito; a concepção universal ou focalizada da APS; e o caráter mais ou menos mercantil da APS, que considera o modelo de intervenção estatal e as relações entre setor público e privado. Esses elementos trazem implicações importantes para o direito à saúde e a constituição de políticas 
mais ou menos equitativas, relacionando-se, portanto, com os padrões de proteção social.

A compreensão da APS não é consensual. São históricas as tensões entre modelos universais e focalizados, e ainda existe uma série de configurações intermediárias, pois os sistemas de saúde no mundo não são constituídos com base em modelos 'puros', e sim por uma mistura de influências (políticas, econômicas e culturais) que atribuem a esses modelos características peculiares.

Por um lado, como expresso na Declaração de Alma-Ata, a APS pode ser interpretada como uma estratégia central para a organização de sistemas de saúde mais equitativos e apropriados às necessidades de saúde da população a que se destinam, respondendo pela maior parte de seus problemas de saúde. Por outro lado, a noção seletiva de APS difundida a partir da Conferência de Bellagio - realizada em 1979, um ano após a Conferência de Alma-Ata - baseia-se na formulação de programas com objetivos focados em problemas específicos de saúde, a fim de atingir grupos populacionais em situação de pobreza (Fausto, 2005).

Alguns argumentos reforçam a importância do caráter universal da APS. O primeiro diz respeito ao seu potencial de reduzir desigualdades em saúde ao favorecer maior equidade. O segundo relaciona-se com sua ação coletiva na prevenção e monitoramento de riscos. O terceiro concerne à intersetorialidade nas ações individuais, familiares e da comunidade. O quarto relaciona-se com valores de democracia e participação social. O quinto e último diz respeito à compreensão da APS como base da organização do sistema de saúde (Fausto e Viana, 2005).

Atualmente, no contexto latino-americano, há diferentes projetos em disputa para a proteção social, os quais podem ser didaticamente resumidos em três linhas principais: ênfase na focalização das políticas sociais restritivas, visando o combate à pobreza; ênfase no universalismo básico, com expansão de serviços sociais básicos nas várias áreas, a fim de cobrir a maior parte da população, e foco em políticas equitativas (Filgueiras et al., 2007); e ênfase na seguridade social, preconizando-se que os direitos e serviços sociais devem ser universais, amplos e destinados a todas as pessoas, independentemente de qualquer outra condição econômica ou social - modelo proposto na Constituição brasileira de 1988. Os diferentes modelos têm implicações importantes para o conjunto das políticas sociais, inclusive para a adoção de uma determinada concepção de APS.

\section{Percurso metodológico}

Com base em contribuições da abordagem sobre proteção social e no estudo de políticas públicas, foram definidas como principais categorias de análise 
da pesquisa: 4 a trajetória da agenda política da Opas; as estratégias e propostas relativas à APS na agenda recente da Opas; e as concepções e significados das propostas de renovação da APS.

Para a caracterização da trajetória e do conteúdo da agenda da Opas, partiu-se da definição de Kingdon (1995) segundo a qual 'agenda' são temas que, em dado momento, mobilizam fortemente a atenção dos dirigentes governamentais e de indivíduos fora do governo, ligados a esses dirigentes. No caso em questão, o foco da análise não era um governo, mas uma agência internacional, atuante na saúde, que busca construir e disseminar uma agenda técnica e política para sensibilizar e influenciar uma audiência de governantes, visando à aceitação pública e à multiplicação de determinadas ideias e propostas (Walt, 1994).

Os métodos envolveram pesquisa bibliográfica e análise documental. Quanto à primeira, realizou-se um levantamento nas bases bibliográficas disponíveis para acesso on-line, incluindo Scientific Electronic Library Online (SciELO), Portal de Periódicos Capes, Bireme e PubMed, com base nos seguintes descritores: atenção primária em saúde, proteção social, agências internacionais, Organização Pan-Americana da Saúde e renovação da atenção primária.

No que tange à análise documental, fez-se uma busca sistemática no sítio oficial da Opas. Foram particularmente valorizados na análise os documentos que tratam do movimento "Renovação da Atenção Primária em Saúde nas Américas", incluindo os relativos ao processo técnico-político de formulação das propostas - declarações, atas de reuniões, comunicados oficiais -, e os principais documentos oficiais da série. Dentre eles, foram selecionados para análise aprofundada os seguintes: documento de posicionamento sobre a renovação da APS nas Américas (Organização Pan-Americana da Saúde e Organização Mundial da Saúde, 2007); documento sobre a formação em medicina orientada para a APS (Organização Pan-Americana da Saúde e Organização Mundial da Saúde, 2008); documento de estratégias para o desenvolvimento de equipes em APS (Organização Pan-Americana da Saúde e Organização Mundial da Saúde, 2009); e documento de redes integradas de serviços de saúde (Organização Pan-Americana da Saúde e Organização Mundial da Saúde, 2010).

A abordagem da análise da retórica (Perelman e Olbrechts-Tyteca, 2000) foi utilizada para a identificação do contexto de formulação dos documentos, dos auditórios a que se destinam e dos principais argumentos da Opas relativos à relevância da APS para os sistemas de saúde contidos em cada um. Além disso, os argumentos e propostas foram estudados à luz das questões e categorias de análise previamente apresentadas, relacionadas às concepções de APS e suas implicações para a proteção social em saúde. 


\section{A atenção primária em saúde renovada na agenda da Opas}

Na década de 1990, a Opas revisitou seus objetivos e estratégias para os seus países-membros. A partir da janela de oportunidades aberta pelo vigésimo quinto aniversário da Declaração de Alma-Ata, na gestão da diretora Mirta Roses Periago (2003), houve uma inflexão política no interior da agência, expressa no lançamento do movimento "Renovação da Atenção Primária em Saúde nas Américas". Com esse movimento, a Opas, além de propor programas específicos no âmbito da APS, sugere a reestruturação dos sistemas de saúde americanos, especificamente os latino-americanos e caribenhos, com base na APS e em valores como direito ao mais alto nível possível de saúde, equidade e solidariedade (Organização Pan-Americana da Saúde e Organização Mundial da Saúde, 2007).

As principais movimentações institucionais no período 2003 a 2010 no interior da Opas para a consolidação da agenda incluíram uma série de declarações, resoluções, relatórios anuais, boletins, revistas e documentos políticos e técnicos. Dentre esses, destaca-se a série de documentos "Renovação da Atenção Primária em Saúde nas Américas", particularmente os quatro selecionados para análise neste estudo (Organização Pan-Americana da Saúde e Organização Mundial da Saúde, 2007, 2008, 2009 e 2010).

O primeiro é o documento de posicionamento Renovação da atenção primária em saúde nas Américas (Organização Pan-Americana da Saúde e Organização Mundial da Saúde, 2007), que apresenta a agenda política da agência relativa ao tema. O documento tem por objetivo realizar recomendações para a renovação da APS e construir coalizões entre os diferentes atores com influência no setor saúde. Destacam-se como principais argumentos: a situação mundial de iniquidades em saúde, a existência de evidências científicas acerca da efetividade e eficácia da APS e a necessidade de resgatar o legado da Conferência de Alma-Ata. O documento apresenta como principal proposta a renovação da APS nas Américas, com o propósito de

Revitalizar a capacidade dos países de elaborar uma estratégia coordenada, eficaz e sustentável para combater os problemas de saúde existentes, prepará-los para novos desafios de saúde e melhorar a equidade. A meta de tal esforço é obter ganhos sustentáveis de saúde para todos (Organização Pan-Americana da Saúde e Organização Mundial da Saúde, 2007, p. 2).

A concepção de APS renovada é bastante genérica e adaptável a diferentes configurações de sistemas de saúde. O documento propõe um modelo de intervenção estatal eminentemente regulador. Tem centralidade na sustentabilidade do sistema de saúde mediante parcerias entre os setores público e privado, como pode ser observado no trecho a seguir: 
a essência da definição renovada da APS é a mesma contida na Declaração de Alma-Ata. No entanto, essa nova definição considera o sistema de saúde como um todo; inclui o setor público, privado e sem fins lucrativos, e aplica-se a todos os países (Organização Pan-Americana da Saúde e Organização Mundial da Saúde, 2007, p. 8).

A agenda técnica e política do movimento de renovação da APS relativa à formação de profissionais de saúde é expressa nos documentos $A$ formação em medicina orientada para a atenção primária em saúde (Organização Pan-Americana da Saúde e Organização Mundial da Saúde, 2008) e Sistemas de saúde com base na atenção primária: estratégias para o desenvolvimento de equipes de atenção primária em saúde (Organização Pan-Americana da Saúde e Organização Mundial da Saúde, 2009). Esses documentos objetivam apresentar diretrizes técnicas para reorientar a formação e/ou qualificação profissional para atuação na APS. Destacam-se como principais argumentos que um sistema de saúde com base na APS deve apoiar-se em recursos humanos adequados e que existe uma dissonância entre a formação e as necessidades de saúde. São propostas reformas curriculares e processos de educação permanente, com foco na formação por competências como estratégia para a qualificação da força de trabalho, por meio de uma abordagem pedagógica programática com ênfase na lógica da produtividade. Há importante fomento às parcerias entre o setor público e o privado como estratégia para a formação e qualificação profissionais.

O quarto documento, Redes integradas de serviços de saúde: conceito, opções politicas e roteiro para a sua implantação nas Américas (Organização Pan-Americana da Saúde e Organização Mundial da Saúde, 2010), apresenta a agenda técnico-política da Opas relativa à organização da rede de serviços de saúde. O texto tem por objetivo analisar a fragmentação dos serviços de saúde e propor um marco conceitual e operacional para a implantação das redes integradas de serviços de saúde (RISS). Parte-se do argumento de que os sistemas de saúde nas Américas caracterizam-se por um alto nível de fragmentação dos serviços, e de que existem evidências científicas que comprovam a ineficiência e ineficácia desse tipo de sistema. O documento traz como proposta a constituição das RISS com foco na articulação do conjunto de provedores, sejam eles públicos ou privados.

Tais redes seriam operacionalizadas por políticas públicas e mecanismos institucionais. A organização da referência e contrarreferência é um dos fatores determinantes para a viabilização de sistemas de saúde universais e abrangentes. Há forte incentivo às parcerias público-privadas no provimento de serviços e, ainda, ao modelo regulador de intervenção estatal.

Esse documento não trata exclusivamente da APS. Contudo, a importância do fato do documento integrar a série "Renovação da APS nas Américas" 
se dá em dois sentidos. Em primeiro lugar, por abordar o debate sobre redes de atenção. Em segundo lugar, por reforçar a ideia de que a APS não se esgota em um nível de atenção, mas é base de estruturação de um sistema de saúde integrado.

O Quadro 1 resume as principais características e argumentos dos documentos analisados.

Quadro 1

\begin{tabular}{|c|c|c|c|c|}
\hline Documento & Contexto & Autoria & Auditório & Principais argumentos \\
\hline $\begin{array}{l}\text { Renovação da atenção } \\
\text { primária em saúde nas } \\
\text { Américas (2007) }\end{array}$ & $\begin{array}{l}\text { Concebido a partir } \\
\text { da janela de } \\
\text { oportunidades aberta } \\
\text { pelas comemorações } \\
\text { do vigésimo quinto } \\
\text { aniversário da } \\
\text { Conferência de Alma- } \\
\text {-Ata e do consenso } \\
\text { internacional } \\
\text { construído com base } \\
\text { na Declaração do } \\
\text { Milênio.* }\end{array}$ & $\begin{array}{l}\text { Elaborado por James Macinko, } \\
\text { conceituado pesquisador do } \\
\text { Departamento de Nutrição, Estudos da } \\
\text { Alimentação e Saúde Pública da } \\
\text { Universidade de Nova York, sob a } \\
\text { coordenação dos especialistas Hernán } \\
\text { Montenegro e Carmen Nebot, ambos } \\
\text { da Área de Organização de Serviços de } \\
\text { Saúde, Tecnologias e Prestação de } \\
\text { Serviços da Opas, sendo o primeiro } \\
\text { consultor de sistemas de saúde e a } \\
\text { segunda, consultora para a proteção } \\
\text { social.Contou com a colaboração de } \\
\text { uma série de especialistas externos à } \\
\text { Opas, dentre eles Barbara Starfield, } \\
\text { referência mundial na temática da APS. }\end{array}$ & $\begin{array}{l}\text { Atores, organizações } \\
\text { e agências tidos } \\
\text { como'neutros' no } \\
\text { processo de } \\
\text { renovação da APS, } \\
\text { a fim de se avançar } \\
\text { na construção de } \\
\text { coalizões, principal- } \\
\text { mente com aqueles } \\
\text { capazes de realizar } \\
\text { investimentos no } \\
\text { setor saúde, sejam } \\
\text { eles públicos ou } \\
\text { privados. }\end{array}$ & $\begin{array}{l}\text { Os desafios epidemiológicos da } \\
\text { atualidade demandam uma } \\
\text { reestruturação dos sistemas e } \\
\text { do cuidado à saúde, a fim de se } \\
\text { promoverem políticas de saúde } \\
\text { apoiadas na equidade. Para tal, } \\
\text { a APS deve ter um caráter } \\
\text { estruturante dos sistemas de } \\
\text { saúde. } \\
\text { Dentre as propostas para se } \\
\text { atingir esse objetivo, } \\
\text { são destacados o } \\
\text { estabelecimento de parcerias } \\
\text { público-privadas e o Estado } \\
\text { regulador como modelo de } \\
\text { intervenção estatal. }\end{array}$ \\
\hline $\begin{array}{l}\text { A formação em } \\
\text { medicina orientada } \\
\text { para a atenção } \\
\text { primária em saúde } \\
\text { (2008) }\end{array}$ & $\begin{array}{l}\text { Elaborado a partir de } \\
\text { uma reunião de } \\
\text { especialistas em } \\
\text { educação médica, } \\
\text { promovida pela Opas } \\
\text { em Belo Horizonte, } \\
\text { MG, em } 2008 \text {. }\end{array}$ & $\begin{array}{l}\text { Coordenado Carmen Nebot (da Área de } \\
\text { Organização de Serviços de Saúde, } \\
\text { Tecnologias e Prestação de Serviços da } \\
\text { Opas) e Carlos Rosales (representação } \\
\text { Opas no Paraguai e consultor de } \\
\text { recursos humanos da Área de Sistemas } \\
\text { e Serviços de Saúde da Opas), com a } \\
\text { participação de Rosa María Borrell } \\
\text { (representação Opas na Argentina e } \\
\text { consultora em educação médica da } \\
\text { Opas), Armando Güemes (consultor de } \\
\text { sistemas e serviços de saúde da Opas) e } \\
\text { José Ruales (representação Opas em El } \\
\text { Salvador e membro do Grupo de } \\
\text { Trabalho em APS da Opas). }\end{array}$ & $\begin{array}{l}\text { Gestores dos níveis } \\
\text { centrais e locais e } \\
\text { instituições e } \\
\text { associações de } \\
\text { ensino. }\end{array}$ & $\begin{array}{l}\text { Um sistema de saúde com base } \\
\text { na APS se apoia em recursos } \\
\text { humanos adequados que } \\
\text { envolvem provedores de } \\
\text { serviços sociais, pessoal } \\
\text { comunitário, gestores, pessoal } \\
\text { administrativo e a população. } \\
\text { Desse modo, propõe-se a } \\
\text { definir competências como o } \\
\text { modo para operar a gestão de } \\
\text { recursos humanos a fim de } \\
\text { estabelecer melhor articulação } \\
\text { entre trabalho, saúde e } \\
\text { educação. }\end{array}$ \\
\hline
\end{tabular}


Continuação - Quadro 1

Sistematização das características e principais argumentos contidos nos documentos analisados

\begin{tabular}{|c|c|c|c|c|}
\hline Documento & Contexto & Autoria & Auditório & Principais argumentos \\
\hline $\begin{array}{l}\text { Sistemas de saúde } \\
\text { com base na atenção } \\
\text { primária: estratégias } \\
\text { para o desenvolvi- } \\
\text { mento de equipes de } \\
\text { atenção primária em } \\
\text { saúde(2009) }\end{array}$ & $\begin{array}{l}\text { Produzido a partir } \\
\text { de uma reunião de } \\
\text { especialistas sobre } \\
\text { as competências } \\
\text { das equipes } \\
\text { multidisciplinares } \\
\text { de APS, promovida } \\
\text { pela Opas durante } \\
\text { o Il Seminário } \\
\text { Internacional de } \\
\text { Atenção Primária - } \\
\text { Saúde da Família } \\
\text { realizado em Fortaleza, } \\
\text { CE, em } 2006 .\end{array}$ & $\begin{array}{l}\text { Coordenado por Rosa María Borrell } \\
\text { representação Opas na Argentina e } \\
\text { consultora em educação médica da } \\
\text { Opas, Charles Godue (representação da } \\
\text { Opas nos Estados Unidos e consultor } \\
\text { de recursos humanos e } \\
\text { desenvolvimento em saúde da Opas) e } \\
\text { Marcelo García Dieguez (consultor de } \\
\text { educação médica da Opas). }\end{array}$ & $\begin{array}{l}\text { Gestores dos níveis } \\
\text { centrais e locais, e } \\
\text { instituições e } \\
\text { associações de } \\
\text { ensino. }\end{array}$ & $\begin{array}{l}\text { A formação médica não é } \\
\text { orientada pelas necessidades } \\
\text { de saúde da população, assim } \\
\text { como não está em consonância } \\
\text { com o propósito da renovação } \\
\text { da APS. Para tal, propõe-se a } \\
\text { promoção de mudanças } \\
\text { curriculares baseadas na } \\
\text { articulação entre ensino e } \\
\text { serviço a fim de qualificar esses } \\
\text { profissionais. }\end{array}$ \\
\hline $\begin{array}{l}\text { Redes integradas de } \\
\text { serviços de saúde: } \\
\text { conceito, opções } \\
\text { políticas e roteiro para } \\
\text { a sua implantação nas } \\
\text { Américas (2010) }\end{array}$ & $\begin{array}{l}\text { Elaborado a partir de } \\
\text { uma série de } \\
\text { consultas aos países- } \\
\text {-membros que } \\
\text { identificaram a } \\
\text { fragmentação dos } \\
\text { serviços de saúde e a } \\
\text { formação profissional } \\
\text { como os grandes } \\
\text { desafios a serem } \\
\text { superados para a } \\
\text { renovação da APS. }\end{array}$ & $\begin{array}{l}\text { A versão preliminar foi escrita por } \\
\text { James Cercone, com revisão } \\
\text { bibliográfica de Eugênio Vilaça Mendes, } \\
\text { ambos consultores da Área de Sistemas } \\
\text { e Serviços de Saúde da Opas. O } \\
\text { documento final foi elaborado por } \\
\text { Hernán Montenegro (Área de } \\
\text { Organização de Serviços de Saúde, } \\
\text { Tecnologias e Prestação de Serviços da } \\
\text { Opas), com colaboração de Eduardo } \\
\text { Levcovitz (representação Opas no } \\
\text { Uruguai), Reynaldo Holder (consultor } \\
\text { da Área de Hospitais e Serviços } \\
\text { Integrais de Saúde da Opas), José } \\
\text { Ruales (representação Opas em El } \\
\text { Salvador e membro do Grupo de } \\
\text { Trabalho em APS da Opas) e Júlio } \\
\text { Soares (consultor da Área de Sistemas e } \\
\text { Serviços de Saúde da Opas e } \\
\text { representação Opas no Chile. }\end{array}$ & $\begin{array}{l}\text { Formuladores de } \\
\text { política, governos e } \\
\text { prestadores de } \\
\text { serviços de saúde, } \\
\text { públicos ou privados. }\end{array}$ & $\begin{array}{l}\text { Parte do pressuposto de que } \\
\text { existe uma fragmentação nos } \\
\text { sistemas de saúde, portanto, } \\
\text { um dos desafios para a } \\
\text { renovação da APS é articulá-la } \\
\text { aos outros níveis de atenção à } \\
\text { saúde. Dentre as medidas } \\
\text { necessárias, destaca-se a } \\
\text { constituição das redes } \\
\text { integradas de serviços de saúde } \\
\text { com foco na articulação do } \\
\text { conjunto de provedores, sejam } \\
\text { eles públicos ou privados. } \\
\text { Propõe ainda uma série de } \\
\text { mecanismos, incluindo } \\
\text { mecanismos de política pública } \\
\text { (jurídicos e não jurídicos) e } \\
\text { mecanismos institucionais } \\
\text { (clínicos e não clínicos) que } \\
\text { viabilizem a rede integrada de } \\
\text { serviços de saúde. }\end{array}$ \\
\hline
\end{tabular}

Fonte: Os autores.

* As oito metas dos objetivos do milênio são: erradicar a pobreza extrema e a fome; atingir o ensino básico universal; promover a igualdade entre os sexos e a autonomia das mulheres; reduzir a mortalidade infantil; melhorar a saúde materna; combater o HIV/AIDS, a malária e outras doenças; garantir a sustentabilidade ambiental; e estabelecer uma parceria mundial para o desenvolvimento (Organização das Nações Unidas, 2000). 
O Quadro 2 resume os resultados da análise dos documentos relativos às relações entre as propostas da APS renovada e a proteção social em saúde, considerando as categorias de análise do estudo.

Quadro 2

\begin{tabular}{|ll|}
\hline Síntese da análise referente às relações entre concepção de APS renovada e a proteção social em saúde \\
\hline Categorias de análise & Concepções presentes na estratégia de APS renovada \\
\hline Visão sobre universalismo/focalização & $\begin{array}{l}\text { Ênfase no universalismo dos serviços de atenção primária à saúde de } \\
\text { qualidade, com foco na equidade. }\end{array}$ \\
Oferta de serviços & $\begin{array}{l}\text { Variável, de acordo com a capacidade institucional dos países - } \\
\text { compatível com vários arranjos. }\end{array}$ \\
Relação entre setor público e privado & $\begin{array}{l}\text { Sistemas mistos, com forte incentivo às parcerias público-privadas e } \\
\text { ênfase na concepção de Estado regulador. }\end{array}$
\end{tabular}

Fonte: Os autores.

\section{Considerações finais}

A saúde é um tema de relevância no cenário internacional em decorrência de suas dimensões social, política e econômica. Em sociedades marcadas por desigualdades sociais, os riscos de adoecer e morrer são distintos nas diferentes classes sociais. Desse modo, a configuração de um modelo de APS abrangente é uma das estratégias necessárias para a garantia e a consolidação do direito à saúde e, consequentemente, para o alcance de níveis mais elevados de qualidade de vida.

A Opas historicamente apresentou continuidades e descontinuidades na trajetória da cooperação técnica para a região das Américas. A partir da gestão de Mirta Roses, em 2003, houve uma inflexão na concepção de APS, traduzida em uma série de documentos que expressam uma agenda política e técnica com foco renovação da APS, na formação e qualificação da força de trabalho orientada à APS e na constituição de uma rede integrada de serviços de saúde.

O conjunto de valores, princípios e elementos centrais que norteiam a proposta de APS renovada expressa, por um lado, uma avaliação negativa de abordagens exclusivamente focalizadas e restritas; e, por outro, as dificuldades para o universalismo que esteja ancorado em uma concepção de seguridade social abrangente e seja efetivado por serviços de saúde públicos estatais, nos diversos níveis de atenção. 
A Opas parece defender posição intermediária de APS com foco no direito aos mais altos níveis possíveis de saúde, equidade e solidariedade. Desse modo, propõe a garantia de um 'básico' social para todos. No entanto, o conceito de básico é fluido e adaptável às diferentes realidades dos países, ou seja, pode incluir uma prestação de serviços mais ou menos ampla e compreender maior ou menor cobertura. Valorizam-se ainda as parcerias entre o setor público e privado, o que pode favorecer uma lógica mercantil nesse e nos outros níveis de atenção à saúde, como é proposto no documento Redes integradas de serviços de saúde (Organização Pan-Americana da Saúde e Organização Mundial da Saúde, 2010). De acordo com Matta (2005), essa posição de estímulo às parceiras público-privadas converge para a agenda proposta pela OMS.

Entre as três perspectivas para a proteção social em tensão na América Latina nos anos 2000 anteriormente mencionadas - a perspectiva focalista, de combate à pobreza; a universalista, vinculada à seguridade social abrangente; e o universalismo básico, relacionado à garantia de serviços sociais básicos de qualidade para a maior parte da população -, o conjunto de propostas e argumentos relativos à APS presente nos documentos da Opas parece mais próximo da concepção do universalismo básico. Tal hipótese, no entanto, merece ser mais bem explorada em estudos posteriores e por meio da análise de casos específicos.

Outro aspecto a ser analisado é a relação dinâmica entre as experiências dos países-membros e a própria configuração da agenda da Opas. Ademais, ao formular a estratégia, a Opas se dirige aos diversos governos das Américas - particularmente aos latino-americanos - buscando sensibilizá-los e formar consensos mínimos em relação à relevância da APS na organização dos sistemas de saúde. Isso explica em grande medida a generalidade das propostas e a sua maleabilidade a diferentes arranjos de sistemas de saúde.

No caso brasileiro, há historicamente uma relação de influências recíprocas entre o país e a Opas (Lima, 2002). Destaque-se que, nos anos 1980, o Brasil foi pioneiro na América Latina no reconhecimento constitucional da saúde como direito de todos e dever do Estado e a primeira nação capitalista da região a adotar formalmente um sistema de saúde público e universal. Nesse contexto, a APS deveria ser organizada sob as diretrizes da seguridade social e do SUS, baseadas em uma noção abrangente de proteção e direito social. No entanto, na prática, a configuração do SUS universal e estatal, assim como da APS, revela numerosas dificuldades e contradições relacionadas a problemas estruturais, como financiamento deficitário e a forte presença do setor privado, o que se reflete nas dificuldades de acesso e na qualidade dos serviços prestados, entre outras questões, fragilizando o direito à saúde.

Ressalte-se que a relevância da garantia de ações e serviços básicos de saúde em termos genéricos constitui uma questão relativamente consensual, 
mesmo diante de distintas concepções de proteção social e de variados arranjos nos sistemas de saúde. Assim, a APS pôde constituir uma agenda em torno do tema conveniente para o esforço de retomada de protagonismo da Opas na difusão de propostas de organização dos sistemas de saúde nas Américas. As repercussões desse movimento nos países-membros, bem como a sua continuidade/descontinuidade no interior da própria Opas, constituem uma agenda profícua para pesquisas futuras.

\section{Colaboradores}

Alessandra Camargo da Silveira realizou a pesquisa, analisou os resultados, concebeu e redigiu a primeira versão do artigo. Cristiani Vieira Machado e Gustavo Correa Matta orientaram o estudo, participaram da discussão dos resultados, da redação e da revisão do artigo. Todos os autores aprovaram a versão final do artigo.

Resumen El artículo trata sobre la agenda de la Organización Panamericana de la Salud para la atención primaria de la salud y sus posibles implicaciones en la protección social en América Latina en los años 2000. El estudio, de naturaleza exploratoria, surgió de la literatura sobre protección social y comprendió como estrategias metodológicas la revisión bibliográfica y el análisis documental, cuya referencia técnica fue el análisis de retórica. La investigación buscó identificar las concepciones y los significados subyacentes a la propuesta de atención primaria de la salud renovada, en lo que concierne a la perspectiva de la protección social, considerando tres aspectos: la población objetivo (universal o focalizada), el alcance (amplio o restringido) y la forma de organización de los servicios (relaciones público-privadas). Los resultados indican que la agenda política de la Organización Panamericana de la Salud presenta históricamente continuidades y discontinuidades. El movimiento de renovación de la atención primaria de la salud manifiesta una inflexión importante en esta agenda, pues sugiere una visión de la atención primaria de la salud como estrategia de restructuración de los sistemas nacionales de salud, más allá del enfoque programático. Sin embargo, en lo que se refiere a la protección social, las propuestas presentadas parecen compatibles con diferentes arreglos de sistemas de salud en lo que atañe a la concepción sobre el universalismo, el alcance de las acciones y las asociaciones público-privadas.

Palabras clave atención primaria de la salud; Organización Panamericana de la Salud; política social; servicios de salud; Américas. 


\section{Notas}

1 Instituto Nacional de Traumatologia e Ortopedia, Ministério da Saúde, Rio de Janeiro, Brasil.

Mestre em Saúde Pública pela Escola Nacional de Saúde Pública Sergio Arouca, Fundação Oswaldo Cruz.

<allecamargo@yahoo.com.br>

Correspondência: Instituto Nacional de Traumatologia e Ortopedia, Gerência de Risco, Avenida Brasil, n. 500, $9^{\circ}$ andar, Caju, CEP 20940-070, Rio de Janeiro, Rio de Janeiro, Brasil.

2 Escola Nacional de Saúde Pública Sergio Arouca, Fundação Oswaldo Cruz, Rio de Janeiro, Rio de Janeiro, Brasil.

Pós-doutora em Ciência Política pela University of North Carolina at Chapel Hill, Carolina do Norte, Estados Unidos, e doutora em Saúde Coletiva pelo Instituto de Medicina Social, Universidade do Estado do Rio de Janeiro.

<cristiani@ensp.fiocruz.br>

3 Escola Nacional de Saúde Pública Sergio Arouca, Fundação Oswaldo Cruz, Rio de Janeiro, Rio de Janeiro, Brasil.

Doutor em Saúde Coletiva pelo Instituto de Medicina Social, Universidade do Estado do Rio de Janeiro.

<gcmatta@fiocruz.br>

4 Este artigo inédito resultou da dissertação Atenção primária em saúde e proteção social: a agenda da Organização Pan-Americana de Saúde nos anos 2000, apresentada por Alessandra Camargo da Silveira com vistas à obtenção do título de Mestre em Ciências na área de Saúde Pública da Escola Nacional de Saúde Pública Sergio Arouca da Fundação Oswaldo Cruz.

\section{Referências}

COSTA, Nilson do R. O Banco Mundial e a política social nos anos 90: A agenda para reforma do setor saúde no Brasil. In: Politicas públicas, justiça distributiva e inovação: saúde e saneamento na agenda social. São Paulo: Hucitec, 1998. p. 125-145.

CUETO, Marcos. O valor da saúde: história da Organização Pan-Americana da Saúde. Rio de Janeiro: Editora Fiocruz, 2007.

FAUSTO, Márcia C. R. Dos programas de medicina comunitária ao Sistema Único de Saúde: uma análise histórica da atenção primária na política de saúde brasileira. 216 p. Tese (Doutorado em Saúde Coletiva) - Instituto de Medicina Social, Universidade do Estado do Rio de Janeiro, Rio de Janeiro, 2005.

FREEMAN, Richard; MORAN, Michael. A saúde na Europa. In: NEGRI, Barjas; VIANA, Ana Luiza d'Ávila (Org.). O Sistema Único de Saúde em dez anos de desafio: o passo a passo de uma reforma que alarga o desenvolvimento e estreita a desigualdade social. São Paulo: Sobravime, 2002. p. 45-64. 
FILGUEIRAS, Fernando et al. Universalismo básico: una alternativa posible y necesaria para mejorar las condiciones de vida. In: MOLINA, Carlos G. (Org.). Universalismo básico/basic universalism: una nueva política social para América Latina. Washington: Banco Interamericano de Desarrollo, 2007. p. 19-55.

KINGDON, John W. Wrapping Things Up. In: KINGDON, John W. Agendas, Alternatives and Public Policies. 2. ed. Boston: Litle Brown, 1995. Cap. 9. p.196-208.

MATTA, Gustavo C. A medida política da vida: a invenção do WHOQOL e a construção de políticas de saúde globais. 226 p. Tese (Doutorado em Saúde Coletiva) - Instituto de Medicina Social, Universidade do Estado do Rio de Janeiro, Rio de Janeiro, 2005.

MATTOS, Ruben A. de. As agências internacionais e as políticas de saúde nos anos 90: um panorama geral da oferta de ideias. Ciência \& Saúde Coletiva, Rio de Janeiro, v. 6, n. 2, p. 377-389, 2001.

LIMA, Nísia T. O Brasil e a Organização Pan-Americana da Saúde: uma história em três dimensões. In: FRINKELMAN, Jacobo (Org.). Caminhos da saúde pública no Brasil. Rio de Janeiro: Editora Fiocruz, 2002. p. 23-116.

ORGANIZAÇÃO DAS NAÇÕES UNIDAS. 2000. Conheça a ONU. Disponível em: $<$ www.onu.org.br/conheca-a-onu $>$. Acesso: Fev. 2010.

ORGANIZAÇÃO PAN-AMERICANA DA SAÚDE; ORGANIZAÇÃO MUNDIAL DA SAÚDE. Renovação da atenção primária em saúde nas Américas: documento de posicionamento da Organização Pan-Americana da Saúde (Opas)/Organização Mundial da Saúde (OMS). Washington: Opas, 2007.
ORGANIZAÇÃO PAN-AMERICANA DA SAÚDE; ORGANIZAÇÃO MUNDIAL DA SAÚDE. La formación en medicina orientada hacia la atención primaria de salud. Washington: Opas, 2008. (Serie La Renovación de la Atención Primaria de Salud en las Américas, 2).

ORGANIZAÇÃO PAN-AMERICANA DA SAÚDE; ORGANIZAÇÃO MUNDIAL DA SAÚDE. Sistemas de saúde com base na atenção primária: estratégias para o desenvolvimento de equipes de atenção primária em saúde. Washington: Opas, 2009. (Série Renovação da Atenção Primária em Saúde nas Américas, 1).

ORGANIZAÇÃO PAN-AMERICANA DA SAÚDE; ORGANIZAÇÃO MUNDIAL DA SAÚDE. Redes integradas de servicios de salud: conceptos, opciones de política y hoja de ruta para su implementación en las Américas. Washington: Opas, 2010. (Série Serie La Renovación de la Atención Primaria de Salud en las Américas, 4).

PERELMAN, Chaïm; OLBRECHTS-TYTECA, Lucie. Tratado da argumentação: a nova retórica. São Paulo: Martins Fontes, 2000.

VIANA, Ana Luiza d'Ávila; FAUSTO, Márcia C. R. Atenção básica e proteção social: universalismo $\mathrm{x}$ focalismo e espaço não mercantil da assistência. In: VIANA, Ana Luiza d'Ávila, ELIAS, Paulo Eduardo M.; IBAÑEZ, Nelson. (Org.). Proteção social: dilemas e desafios. São Paulo: Hucitec, 2005. p. 150-167.

VIANA, Ana Luiza d'Ávila; SILVA, Hudson P. da; ELIAS, Paulo Eduardo M. Saúde e desenvolvimento. Ciência \& Saúde Coletiva, Rio de Janeiro, v. 12, supl., p. 1.765-1.776, 2007.

WALT, Gill. The International Arena: Who Is Driving Policy? In: WALT, Gill. Health Policy: An Introduction to Process and Power. Londres: Zed Books, 1994. Cap.7. p. 122-152.

Recebido em 12/08/2013

Aprovado em 19/09/2013 